\title{
Quaderni
}

QUADERNI Communication, technologies, pouvoir

89 | Hiver 2015-2016

Penser l'avenir : le CESTA, un think tank atypique

\section{Yves Stourdzé et les réalisations du CESTA}

\section{Brigitte Chamak}

\section{(2) OpenEdition \\ Journals}

Édition électronique

URL : http://journals.openedition.org/quaderni/952

DOI : 10.4000/quaderni.952

ISSN : 2105-2956

Éditeur

Les éditions de la Maison des sciences de l'Homme

Édition imprimée

Date de publication : 5 janvier 2016

Pagination : 11-24

Référence électronique

Brigitte Chamak, "Yves Stourdzé et les réalisations du CESTA », Quaderni [En ligne], 89 | Hiver 2015-2016, mis en ligne le 05 janvier 2018, consulté le 20 avril 2019. URL : http://

journals.openedition.org/quaderni/952; DOI : 10.4000/quaderni.952 


\section{Dossier}

\section{Yves Stourdzé et les réalisations du CESTA}

Dans les années 1980, le CESTA(Centre d'étude des systèmes et technologies avancées) était un véritable organisme de conseil de la présidence de la République, impliqué dans la mise en œuvre des suites du sommet de Versailles des chefs d'État et de Gouvernement des sept pays industrialisés et dans l'élaboration du projet de coopération technologique, Eurêka. De 1983 à 1986, Yves Stourdzé en assura la direction générale. Le rapport de François Mitterrand (« Technologie, emploi et croissance ») présenté au sommet de

\section{Brigitte} Chamak

Université Paris 5

CERMES3

(Centre de Recherche Médecine,

Santé, Santé Mentale, Société)

INSERM U988 - CNRS - EHESS
Versailles le 5 juin 1982 proposait de réaliser « une mobilisation sans précédent du capital vers l'industrie et la recherche ${ }^{1} »$ et de construire une nouvelle civilisation. Le président cherchait à lancer un programme concerté de croissance sélective par la technologie, à accorder une priorité à l'emploi et aux conditions de travail, et à favoriser l'épanouissement des cultures via les nouveaux outils de communication. Il s'agissait de travailler à un « Nouvel Ordre technologique mondial ${ }^{2} »$, perçu comme le moyen de sortir de la « crise ».

La crise économique des années soixante-dix et la volonté d'adaptation des grandes entreprises, qui entendaient diminuer leurs coûts en réduisant le nombre de leurs salariés et en automatisant leur activité, avaient provoqué une montée du chômage. Les divisions au sein de la droite, le poids du chômage et celui de la crise économique conduisirent à un changement de majorité aux élections de 1981. Écartée du pouvoir pendant un quart de siècle, la gauche annonçait un vaste programme de réformes de structures (décentralisation, nationalisations et planification) et donnait la priorité à la lutte contre le chômage et à la relance de l'activité économique. Les nationalisations vi- 
saient à placer entre les mains de l'État l'essentiel du crédit et des secteurs clés de la production. Toute une série de mesures étaient destinées à modifier le visage de la société française pour éviter les tentations de repli sur soi et favoriser l'adaptation aux mutations technologiques. La création du CESTA et le colloque national « Recherche et Technologie » (janvier 1982) se situent dans ce contexte, le gouvernement ayant décidé de faire de la recherche et du développement technologique une priorité nationale.

La préhistoire du CESTA est à retrouver dans les échanges entre scientifiques et politiques dans les années 1970, et notamment au sein du Groupe des Dix ${ }^{3}$, créé en 1968 à l'initiative de Jacques Robin (médecin), Edgar Morin (sociologue), Henri Laborit (biologiste) et Robert Buron (homme politique). La première partie de cet article traitera de cette préhistoire et du rôle de Jacques Attali, membre du Groupe des Dix dans les années 1970 et conseiller spécial du président François Mitterrand de 1981 à 1991. La seconde partie analysera l'implication d'Yves Stourdzé et du CESTA dans l'animation, et le suivi du groupe de travail « Technologie, Croissance, Emploi »(TCE), créé au lendemain du Sommet de Versailles, et la conception du projet EUREKA. Enfin, sera abordé le rôle du CESTA dans le développement des sciences cognitives en France.

\section{Préhistoire du CESTA}

À la fin des années 1970 et sous l'impulsion de Claude Alphandéry ${ }^{4}$ se retrouvèrent chez ce dernier, une dizaine de samedis de suite, Jacques Attali, Edmond Maire, Jacques Robin,
Michel Rocard, René Passet et le sociologue et économiste Robert Fossaert ${ }^{5}$. L'objectif était de réfléchir aux problèmes économiques, politiques, sociaux, démographiques, scientifiques, techniques et de se préparer à un éventuel succès de la gauche aux élections. Conseiller de François Mitterrand, Jacques Attali était chargé de préparer des notes sur ces différents sujets.

Ces rencontres prolongeaient d'une certaine façon celles du Groupe des Dix qui ont eu lieu entre 1969 et 1976 entre scientifiques de différentes disciplines et personnalités politiques d'orientation socialiste avec pour but « de déterminer ce que signifierait pour la politique la prise en compte des nouvelles données des sciences $d u$ vivant, de la bio-anthropologie, de la connaissance des comportements, de la cybernétique, de la théorie de l'information et de la théorie des systèmes ${ }^{6}{ } »$. L'objectif était d'intensifier les relations entre sciences et politique afin de pallier le «côté magique de la décision politique ${ }^{7} »$. Ces rencontres se proposaient d'élaborer un nouveau système d'interprétation, « une nouvelle grille de référence ». La première période du Groupe des Dix se caractérise par la volonté d'élaborer un projet politique et une théorie de l'organisation de la société fondée sur une meilleure utilisation des connaissances scientifiques en vue de construire une société plus égalitaire. Des personnalités scientifiques extérieures ont été invitées à parler de leurs livres ou de leurs travaux. En 1971, c'est Joël de Rosnay qui a été invité à présenter un exposé intitulé Vers une société en temps réel, expliquant comment la révolution de la communication et de l'ordinateur allait changer notre façon de travailler, de réfléchir, d'accéder à l'information. Intéressé par les discussions et 
les échanges au sein du groupe, il a demandé à en faire partie. C'est lui qui en décembre 1981 rédigera le rapport présentant les objectifs, les propositions d'organisation, de fonctionnement et la mise en œuvre du CESTA.

Jacques Robin joua également un rôle important dans la création du CESTA. En 1979, il se rendit aux États-Unis et au Japon pour participer à des débats sur les perspectives technologiques de l'Institut Stanford et évaluer les projets de l'industrie pharmaceutique japonaise. Dès son retour il rencontra Jacques Attali qui lui aurait confié que si François Mitterrand était élu, il tenterait de recréer « un Groupe des Dix proche du pouvoir » afin de mettre en place « une cellule où des intellectuels, des scientifiques, des chercheurs et des politiques pourraient en permanence réfléchir ensemble ${ }^{8} »$.

Après l'élection de François Mitterrand à la présidence de la République en 1981, Jacques Attali proposa son projet de transformer l'Institut Auguste Comte ${ }^{9}$, fondé par Valéry Giscard d'Estaing en 1977 et réservé à un nombre limité de personnes, essentiellement des polytechniciens, en un centre plus ouvert, le CESTA, où certains anciens membres du Groupe des Dix pourraient se retrouver et participer à la réalisation d'un projet nouveau axé sur des échanges accrus entre industriels, scientifiques, syndicalistes, organismes de recherche, collectivités et entreprises. Le CESTA, situé sur la Montagne Sainte-Geneviève à Paris au ministère de la Recherche et de la Technologie, a été créé dans l'optique d'orienter les choix de financement des projets scientifiques et techniques et avait pour mission l'aide à la décision et la prospective technologique mais aussi l'information et la formation du plus grand nombre pour une diffusion des nouvelles technologies.

L'histoire du CESTA est indissociable du contexte de concurrence technologique internationale. Les États-Unis avaient pris le premier rang mondial en matière de recherche scientifique et technique et les Japonais avaient annoncé en 1981 leur projet « $5^{\mathrm{e}}$ génération 》 d'ordinateurs qui proposait de réaliser des ordinateurs « intelligents » capables de prendre des décisions. La bataille informatique faisait rage. Le CESTA joua le rôle de catalyseur favorisant la mise en œuvre d'une coopération technologique européenne de grande ampleur.

La décision de la création du CESTA a été prise lors du Conseil des ministres du 4 novembre 1981. À la demande du Premier ministre Pierre Mauroy, Joël de Rosnay, alors directeur des Applications de la Recherche à l'Institut Pasteur, a été chargé de proposer un projet. Avec l'aide de François de Lavergne, Chef du Groupe de Prospective au ministère de l'Urbanisme et du Logement, Jean-Claude Derian, Conseiller du Président de l'Agence Coopération et Aménagement et Thierry Gaudin, Chef du Centre de prospective et d'Évaluation auprès du ministère de la Recherche et de la Technologie, Joël de Rosnay rédigea en décembre 1981 un rapport présentant les objectifs, les propositions d'organisation, de fonctionnement et la mise en œuvre du CESTA. Ce centre était conçu comme un outil privilégié pour construire un projet de société centré sur les nouvelles technologies : "le CESTA veut contribuer par ses missions et ses fonctions à l'enrichissement d'une culture capable de sous- 
tendre un projet de société10 ». Les objectifs étaient de faire du CESTA un « organisme d'étude et de formation qui a pour vocation d'apporter sa contribution au développement et à la diffusion des technologies, tout en analysant et en préparant les conditions de leur insertion dans les structures socio-économiques et socioculturelles de la société française ${ }^{11} »$. Ce centre avait pour mission de constituer un observatoire du développement technologique, un lieu de veille, de prévision et de prospective, un support à la décision publique et privée, un carrefour d'animation et d'information sur les technologies de pointe et leurs conséquences économiques, sociales et politiques. Il s'agissait « d'éclairer les décisions stratégiques et politiques dans l'esprit de l'Office of Technology Assessment aux États$\mathrm{Unis}^{12} »$.

Jacques Attali ayant demandé à Jacques Robin de s'occuper de la mise en place du CESTA, ce dernier accepta sous réserve de ne s'engager que pour une courte période. Estimant que la culture était un élément important, il proposa à Joël de Rosnay d'inclure dans son rapport sur le CESTA une annexe concernant la création d'un Groupe Science-Culture, dans la suite du Groupe des Dix, qui travaillerait en étroite collaboration avec le Centre Mondial de Valorisation Sociale de la Micro-Informatique. Avec Henri Atlan et Jean-Pierre Dupuy, il rédigea un texte qui devint l'annexe $\mathrm{n}^{\circ} 11 \mathrm{du}$ rapport CESTA. Les missions fixées par ce groupe étaient des missions de recherche sur le thème de la complexité et de l'autonomie (logique, épistémologie et philosophie) mais aussi des missions de formation et de sensibilisation d'un large public sous forme de forums, de débats, et de séminaires.
En avril 1982, un document produit par la délégation résumait les missions du CESTA. Une fonction centrale, l'assistance aux choix technologiques, était prise en charge par deux groupes : le groupe d'études et d'analyses stratégiques des filières technologiques, axé sur la prévision technologique, confié à Yves Stourdzé et le groupe de recherches sur l'insertion des techniques et leur impact socio-culturel, dirigé par François de Lavergne.

L'association CESTA fonctionna pendant dix-huit mois puis, en juillet 1983, le CESTA devint un EPIC, Établissement Public à caractère Industriel et Commercial. Yves Stourdzé en assura la direction générale. La nomination d'Yves Stourdzé a été riche en péripéties car la concurrence à la direction de ce centre était élevée. Yves Stourdzé avait le handicap de ne pas sortir d'une grande École, mais il bénéficiait de l'estime de Jacques Attali et de Jacques Dondoux, directeur général des Télécommunications. Avec Yves Stourdzé, le CESTA devint un véritable organisme de conseil de la présidence de République, impliqué dans l'organisation et le suivi du Sommet de Versailles, puis dans l'élaboration du projet de coopération technologique Eurêka.

\section{Rôle du CESTA dans l'élaboration d'une politique de coopération technologique euro- péenne}

Le CESTA prit en charge l'animation et le suivi du groupe de travail Technologie, Croissance, Emploi (TCE), créé au lendemain du sommet de Versailles pour réfléchir à l'élaboration d'un programme de croissance par la coopération technologique. Dès 1982, Yves Stourdzé en assura le 
secrétariat général. Dans une lettre du 9 août 1982, il écrivait : "Les chefs d'État et de gouvernement réunis au Sommet de Versailles à l'invitation du président de la République française ont décidé la création d'un groupe de travail « Technologie, Croissance, Emploi » chargé de réfléchir à un programme concerté de croissance par le coopération technologique. Cette commission doit remettre son rapport au 31/12/82. Le président de la République a demandé à Jacques Attali, conseiller spécial, d'en assurer la présidence. Le professeur François Gros, conseiller du Premier ministre pour les questions scientifiques, l'assiste dans cette tâche. J'assure le secrétariat général du groupe de travail ${ }^{13} »$. Après s'être réuni sept fois, ce groupe proposa 18 projets de coopération internationale. Le rapport, paru en janvier 1983, « se concentre sur des problèmes auxquels la science et la technologie peuvent offrir des solutions, sans que ces dernières ne constituent pour autant de quelconques panacées ${ }^{14} »$. L'accent est mis sur « le rôle que peuvent jouer la science et la technologie dans l'amélioration de la croissance économique et de la situation de l'emploi, ainsi que dans la promotion de la culture et de l'éducation ${ }^{15} »$. Les 18 projets sont regroupés en quatre thèmes : 1) améliorer les conditions de vie et d'emploi et protéger l'environnement (trains à grande vitesse, microélectronique, informatique, robotique, biotechnologies, impact des nouvelles technologies sur les industries traditionnelles, l'éducation et la formation professionnelle, acceptabilité des nouvelles technologies); 2) stimuler les conditions de la croissance par une meilleure gestion des sources d'énergie (énergie solaire photovoltaïque, fusion thermonucléaire, photosynthèse, réacteurs à neutrons rapides) ; 3) assurer une meilleure gestion des ressources alimentaires (technologies alimentaires, aquaculture) ; 4) promouvoir des progrès généraux en recherche fondamentale (biologie, physique des hautes énergies, exploration du système solaire $)^{16}$. Enseignement assisté par ordinateur, conception assistée par ordinateur, traduction assistée par ordinateur, images électroniques sont présentés comme des priorités dans le domaine de l'éducation et de la formation. L'ordinateur se trouve au centre des transformations envisagées et l'acceptabilité sociale du changement technique figure comme un élément crucial. Il s'agit de financer des projets de coopération entre différentes entreprises européennes afin de se regrouper et empêcher ainsi un monopole, notamment pour les technologies de l'information et de la communication. Le groupe de travail TCE se veut une réponse européenne au mouvement de déréglementation qui affecte en premier lieu les grands réseaux économiques : industrie de service, activités bancaires et financières, systèmes stratégiques. Il analyse les nouvelles données mondiales, les enjeux majeurs et indique des voies de coopérations européennes. Il initie un mode de négociation internationale dans le champ de la technologie pour un nouveau partage des marchés et des zones d'influence ${ }^{17}$.

En mars 1985, François Mitterrand, irrité par les pratiques américaines d'injonction à participer à l'initiative de défense stratégique ${ }^{18}$ (IDS), chargea ses conseillers, Jacques Attali et Pierre Morel, d'élaborer des propositions et c'est le CESTA qui fut chargé de concevoir un projet de coopération européenne orienté vers la recherche civile ${ }^{19}$. Début avril, le ministre français des Relations Extérieures, Roland Dumas, lança sa proposition de créer une « European Research Coordination 
Agency » mais en mai au sommet de Pittsburg, la proposition est modifiée devant la réaction réservée des partenaires européens à ce projet qui apparaissait comme une prise de position commune des Européens face à l'IDS. Abandonnant l'idée d'une agence, Eurêka est transformé en une simple action ayant pour but de favoriser une coopération européenne à visée civile. Entre mars et juin 1985, Yves Stourdzé et son équipe rédigèrent le livre blanc, La Renaissance technologie de l'Europe, dans le cadre du lancement du programme Eurêka de coopération technologique européenne. Ces propositions françaises, axées sur cinq grands thèmes : l'informatique, la robotique, les télécommunications, les biotechnologies et les matériaux, furent présentées les 28 et 29 juin 1985 au Conseil Européen de Milan, qui apporta son soutien au projet français Eurêka visant à créer une Europe de la technologie ${ }^{20}$. Cet accord acté aux Assises européennes de la technologie du 17 juillet à Paris, constitua l'acte de naissance d'Eurêka ${ }^{21}$.

Alors même que le CESTA avait joué un rôle central dans la conception des projets politiques de coopération technologique internationale et européenne, Yves Stourdzé se trouva déchargé du suivi d'Eurêka. C'est Yves Sillard, qui de 1976 à 1982 avait assuré la direction générale du Centre National d'Études Spatiales sous la présidence d'Hubert Curien, qui fut nommé pour mener à bien ce projet. Un groupe de représentants des dix-huit pays prépara les décisions de la conférence ministérielle d'Hanovre des 5 et 6 novembre 1985 et la Charte d'Eurêka fut adoptée : «Eurêka a pour objectif, par le renforcement de la coopération entre les entreprises et les instituts de recherche dans le domaine des hautes technologies, d'accroître la productivité et la compétitivité des industries et des économies nationales européennes sur le marché mondial et de contribuer ainsi au développement de la prospérité et de l'emploi : Eurêka doit permettre à l'Europe de maîtriser et d'explorer les technologies importantes pour son avenir et de développer ses capacités dans des secteurs essentiels $^{22} »$. Dix projets concrets sont approuvés lors de la conférence : microordinateur domestique, calculateur vectoriel compact, fabrication de silicium amorphe, robot pour le textile, réalisation de membranes filtrantes, laser, repérage des composants polluants dans la stratosphère, réseau de recherche européenne, tests de diagnostic pour maladies sexuellement transmissibles, atelier optronique. Lors de sa présentation à la journée du 17 octobre 1985 organisée par l'Observatoire international des images, Michel Feldmann, l'un des adjoints d'Yves Stourdzé, définissait Eurêka comme « un programme finalisé pré-commercial constitué d'un petit nombre d'actions de grande ampleur $^{23} »$. Il citait cinq thèmes : Euromatique (informatique, microélectronique, intelligence artificielle) ; Eurobot (système de production intégrée, robotique de $3^{\mathrm{e}}$ génération) ; Eurocom (télécommunication), Euromat (matériaux nouveaux) et Eurobio (biotechnologies).

Le CESTA continua à animer cinq des 18 actions de coopération internationale lancée par le groupe TCE : robots autonomes multiservices, réseau international des biotechnologies, impact des nouvelles technologies sur les industries traditionnelles, nouvelles technologies appliquées à l'éducation, formation professionnelle et culture. Soucieux de l'attitude du public à l'égard des nouvelles technologies, le CESTA organisa force 
manifestations, expositions et démonstrations.

\section{Rôle du CESTA dans le développement des sciences cognitives en France}

Dès novembre 1982, le CESTA, en collaboration avec l'AFCET, Association Française des sciences et technologies de l'information et des systèmes, regroupant essentiellement des informaticiens et des chercheurs en informatique, organisa des Assises pour le développement des sciences de l'organisation ${ }^{24}$. Le compte-rendu des Assises $^{25}$, publié en décembre 1982 par le CESTA débutait par un historique : «L'organisation a connu ses grandes heures après la Seconde Guerre mondiale : Organisation Scientifique du Travail (OST), dans les années 50, techniques quantitatives (notamment Recherche Opérationnelle) dans les années $60 »$, puis dressait un constat : «La France a cherché à transposer, de façon un peu mécaniste, le modèle américain de la décentralisation et du contrôle aux grands groupes français, en lui attribuant une valeur universelle [...]. On a en fait transposé l'apparence et non la réalité. » $\mathrm{Il}$ est recommandé de poursuivre « une recherche de l'efficacité, une modification des aspirations et transformations des valeurs de l'individu ».

L'organisation de colloques visait à souder les professionnels de ces domaines cruciaux que sont l'électronique, l'informatique et la robotique. Le premier colloque organisé par le CESTA, la semaine internationale de l'image électronique, eut lieu à Biarritz du 21 au 25 mai 1984, et profita de l'inauguration du réseau expérimental interactif à large bande des télécommunications françaises. Cette manifestation organisée par Michel
Feldmann et Jean-Paul Gillet ${ }^{26}$ a connu un grand succès (près de mille personnes y participèrent). Reconnaissance de formes, reconstruction médicale, télédétection, codages, robotique, synthèse d'images, vidéocommunication, figuraient parmi les sujets traités. Ces thèmes ont été approfondis plus tard dans les colloques en sciences cognitives dont le CESTA a favorisé l'éclosion, en organisant notamment, en juin 1985, le premier colloque en sciences cognitives en France, Cognitiva 85 : de l'intelligence artificielle aux biosciences. Dans le procès-verbal du conseil d'administration du CESTA du 11 septembre 1984, il est fait mention que : «Jacques Robin insiste sur l'extraordinaire croissance de l'intérêt pour les sciences cognitives aux États-Unis. Il pense que nous aurons des difficultés à suivre en France cette dynamique et qu'il faut regarder avec beaucoup d'intérêt les applications de l'intelligence artificielle et des systèmes experts ${ }^{27} \gg$. Michel Feldmann, co-organisateur du colloque avec Françoise Fogelmann et Norbert Cot, raconte comment un jour Yves Stourdzé, revenant du Japon, lui a demandé de se charger d'organiser un colloque qui aurait pour nom Cognitiva et qui se trouverait à la croisée des chemins entre intelligence artificielle et neurosciences. «Ce colloque a eu un très grand succès : le rapprochement entre informatique, psychologie, neurobiologie est apparu comme très original à l'époque ${ }^{28} »$. L'objectif était de mettre en commun différentes compétences pour mieux comprendre comment fonctionnent la pensée, la mémoire, le langage, l'apprentissage, la perception, dans le but de modéliser et simuler ces facultés cognitives. Communication homme/ machine, traitement d'images, reconnaissance de formes, systèmes experts, représentation des connaissances, robotique, étaient autant de sujets 
traités au cours de ce colloque. Le président du comité scientifique du colloque était JacquesLouis Lions de l'INRIA (Institut National de la Recherche en Informatique et Automatique). La centaine de communications et la vingtaine de posters présentés relevaient de l'intelligence artificielle. Alan Newell, l'un des pionniers de l'intelligence artificielle, donna une conférence inaugurale sur le concept d'architecture en informatique qu'il généralisait à la cognition humaine. Il présenta le système Soar, système « intelligent » de résolution de problèmes qui « apprend à partir de ses expériences ». Ainsi dès leur promotion par le CESTA, les sciences cognitives étaient définies d'emblée comme une science orientée vers les pratiques et concepts élaborés en intelligence artificielle ${ }^{29}$. Le CESTA organisa ensuite des Séminaires Cognitiva traitant d'intelligence artificielle, en particulier de systèmes experts. Le milieu professionnel convoqué était composé en majorité d'informaticiens, de chercheurs de l'INRIA, du CNET, du CNRS, membres de l'AFCET et/ou de l'ARC (Association pour la Recherche Cognitive). Un an après le colloque sur les sciences cognitives, le CESTA édita un livre sur l'intelligence artificielle ${ }^{30}$. Yves Stourdzé en rédigea l'avant-propos : «L'Intelligence Artificielle nous ouvre les portes féériques des connexions démultipliées à l'infini. Elle nous introduit dans un jeu où l'algèbre de la « conception " trouve " naturellement " sa transcription géométrique dans la réalisation. C'est la matérialisation de toute pensée dès lors que celle-ci se formalise. Machines et réseaux intelligents : notre univers se métamorphose. Sa gestion, tant civile que militaire, en sera totalement transformée. Désormais ce qui peut s'imaginer peut se faire. Et ce qui peut s'imaginer, c'est précisément ce qui peut se calculer. » Pour Yves Stourdzé, l'intelligence artificielle était une révolution et il s'adressait à «tous ceux qui ne voulaient pas se retrouver demain dépassés face à cette révolution, vieillis dans un monde où les machines rêvent... ». Les méthodes en intelligence artificielle ont peu à peu envahi différents domaines scientifiques et les chercheurs du laboratoire de dynamique des réseaux (LDR), situé sur le même lieu que le CESTA, ont inauguré une démarche, celle de l'usage des techniques nouvelles issues de la théorie des automates développés sous le nom de connexionnisme (modélisation de phénomènes mentaux ou comportementaux à l'aide de réseaux d'unités simples interconnectées nommées réseaux neuronaux).

Sur le site du CESTA, rue de la Montagne SainteGeneviève, le Groupe Science/Culture, association régie par la loi 1901, comptait trois départements : 1) le LDR, orienté vers des recherches sur la formalisation et la simulation des systèmes complexes ; 2) le CREA, centre de recherche en épistémologie et autonomie ${ }^{31}$; 3) le GRI, groupe de réflexions interdisciplinaires, qui se présentait comme un prolongement du Groupe des Dix.

Le LDR et le CREA jouèrent un rôle majeur dans l'épanouissement des sciences cognitives en France. Le LDR était un laboratoire orienté vers la modélisation mathématique de systèmes physico-chimiques et biologiques. Henri Atlan, Maurice Milgram (informaticien) et Gérard Weisbuch (physicien) s'intéressaient à la dynamique des réseaux et Françoise Fogelman a été la première informaticienne à travailler sur la modélisation des réseaux de neurones (connexionnisme). Les méthodes mises au point au LDR ont été utilisées 
par certains chercheurs du CREA. Le CREA, l'un des trois laboratoires en sciences sociales de l'École polytechnique, s'est intéressé au statut épistémologique des modèles de la complexité et de l'auto-organisation en biologie mais aussi en sciences sociales, et plus particulièrement en économie. Les questions sur l'auto-organisation avaient fait l'objet d'un colloque international organisé en 1981 à Cerisy. C'est au cours de ce colloque que furent conçues les bases théoriques du CREA et du LDR. Ancien élève de polytechnique, Jean-Pierre Dupuy a fondé le CREA avec Jean-Marie Domenach qui voulait en faire le centre de recherches en épistémologie de l'École polytechnique. Pendant trois ans, Jean-Pierre Dupuy organisa une formation intensive en logique. Le logicien Daniel Andler intégra le CREA, ainsi que d'autres logiciens, mathématiciens et philosophes : Dan Sperber, François Récanati, Pierre Jacob, Pascal Engel, Joëlle Proust. Tous participèrent à l'essor des sciences cognitives et au développement de la philosophie analytique en France.

\section{Fin du CESTA}

Les changements occasionnés par le gouvernement de Laurent Fabius (1984-1986), et sa gestion pragmatique des affaires au détriment des grands objectifs idéologiques des débuts du septennat, vont jouer un rôle dans la chute du CESTA. En prise directe avec les milieux politiques, ce centre n'a pas échappé aux tensions et rivalités. Des doutes quant à la rentabilité du CESTA ayant été formulés, le Cour des Comptes en examina la gestion en 1984. Constatant que le CESTA avait reçu de l'État près de 24 millions de francs pour l'année 1984, le premier président de la Cour des
Comptes, André Chandernagor, annonça dans un courrier du 2 avril 1985 de « graves anomalies dans sa gestion et son fonctionnement ${ }^{32} »$. Jugeant que le personnel permanent était trop nombreux par rapport aux prévisions faites au début de sa création, il critiqua les orientations prises : « Il apparaît en définitive que le CESTA se cantonne essentiellement dans le domaine qui lui est le plus commodément abordable, celui de la " mise en réseau "d'interlocuteurs issus d'horizons différents. Il est ainsi conduit à mettre l'accent sur l'organisation de colloques, de séminaires, la présentation de matériel pédagogique. » Les multiples attaques contre le CESTA ont porté leurs fruits alors même que le travail réalisé en peu de temps a abouti à la conception du projet Eurêka et au développement des nouvelles technologies de l'information et de la communication. Le fait même que le projet Eurêka accepté par les pays européens fut retiré au CESTA montre la puissance des zones d'influence et la volonté d'affaiblir la structure que Jacques Attali et Yves Stourdzé avaient contribué à mettre en place. Fin 1985, des examens révélèrent à Yves Stourdzé un état de santé très préoccupant. En décembre 1986, il mourut à l'âge de 39 ans. Aussitôt après sa mort, le budget du CESTA fut amputé d'un quart et ses effectifs passèrent de 80 à 60 salariés.

Les élections du 16 mars 1986 donnèrent une légère victoire à la droite, ouvrant une période de cohabitation entre un président de gauche et une majorité de droite. Jacques Chirac, chef de la nouvelle majorité, fut nommé Premier ministre. Un décret du 17 novembre 1987 ordonna la dissolution du CESTA au $1^{\text {er }}$ janvier $1988^{33}$. Par un courrier daté du 29 janvier 1987, Jacques Chirac demandait à Roger Martin, ancien président de 
Saint-Gobain Pont-à-Mousson et de l'Institut Auguste Comte, un rapport sur la vocation du site de la Montagne Sainte-Geneviève ${ }^{34}$. Roger Martin réaffirma que ce site était voué à la formation et à la recherche de haut niveau pour une élite.

\section{Vie et mort d'un organisme d'État}

Entre 1982 et 1985, les réalisations du CESTA ont été impressionnantes. Le travail, la rapidité d'exécution et les idées visionnaires d'Yves Stourdzé et de son équipe forcent l'admiration. Qu'en si peu de temps ait été élaboré un projet de coopération technologique européenne accepté par les États membres de l'époque, qui perdure toujours, relevait de la gageure. Pourtant, le CESTA a disparu et Yves Stourdzé ne lui a pas survécu. Par contre, le LDR et le CREA ont continué leurs réflexions et leurs recherches en philosophie analytique et les travaux en sciences cognitives sur le site de la Montagne-Sainte-Geneviève, ont constitué un noyau structurant autour de philosophes, logiciens, mathématiciens, neurophysiologistes, chercheurs en informatique, en linguistique et en psychologie, et ont favorisé le développement des sciences cognitives en France ${ }^{35}$.

Depuis les années 1990, les recherches sur l'élaboration des politiques publiques s'intéressent tout particulièrement aux think tanks (« boîtes à idées »), cette organisation de la société civile qui se donne pour mission d'éclairer, voire d'influencer, les citoyens, les élus ou les groupes de pression au sujet des grandes questions de politique publique $^{36}$. Ni think tank ni collège invisible ${ }^{37}$, le Groupe des Dix peut être décrit comme un cercle de réflexion informel qui a joué un rôle non négligeable dans la vie politique française.
Certains de ses membres ont influencé les décisions prises dans les années 1980 et, en particulier l'orientation vers le projet d'une société centrée sur les nouvelles technologies. La création du CESTA est directement liée à l'influence de Jacques Attali, qui lui-même a puisé, dans les échanges au sein du Groupe des Dix, les idées dont il allait se servir pour conseiller le président la République. Au moment de l'arrivée de la gauche au pouvoir, les liens qui s'étaient créés entre certains membres, dont Jacques Attali, Jacques Robin et Joël de Rosnay, allaient permettre la concrétisation de la création d'une structure originale, à la fois observatoire du développement technologique, lieu de veille, de prévision et de prospective, support à la décision publique et privée, carrefour d'animation et d'information sur les nouvelles technologies et leurs conséquences économiques, sociales et politiques.

Le travail réalisé au CESTA a donné l'opportunité au président de la République de réagir rapidement à la proposition de Ronald Reagan de participer à l'IDS en 1985. Il a pu présenter une alternative qui a reçu l'assentiment des autres pays européens et c'est ainsi que le projet Eurêka a vu le jour. L'histoire du CESTA est particulièrement intéressante pour ceux qui se penchent sur l'analyse de l'État en action et l'influence des idées d'un groupe d'intellectuels mais aussi pour ceux qui voudraient étudier comment, en France, les nouvelles technologies se sont développées et diffusées dans les années 1980, avec les bouleversements dont nous observons les conséquences aujourd'hui. Contrairement à ce que prônait François Mitterrand dans son rapport au sommet de Versailles le 5 juin 1982, 
les biotechnologies n'ont pas véritablement fait reculer la faim, la maladie et la surpopulation. Et si l'électronique et la robotique ont multiplié la capacité de production et de création de nos économies, celles-ci n'ont pas permis la réduction du chômage mais au contraire son aggravation. Il est vrai qu'avec les nouvelles technologies de la communication, une autre forme de civilisation s'est instaurée mais avec de nouveaux problèmes, de nouvelles formes de violence, d'égoïsme, de déséquilibres et de conflits.

Dans son livre, Technique et idéologie. Un enjeu de pouvoir ${ }^{38}$, Lucien Sfez a interrogé les discours sur la technique. Pour lui, un discours, une prise de position politique n'ont d'efficacité que s'ils reposent sur une fiction. De même, les technodiscours n'ont de résonance dans la société que parce qu'ils reposent sur des narrations, des fictions, au sens de mode de légitimation spécifique d'un type de discours, et de l'ordre social que ces discours produisent en même temps qu'ils en sont le produit. Ces fictions de la technique ont un fort contenu idéologique et utopique. Les décisions sociopolitiques se réfèrent à la technique pour acquérir leur légitimité et faire apparaître la décision comme le résultat d'un processus objectif. De même le développement technologique puise dans le sociopolitique sa justification au nom du Progrès de la Société et de l'Humanité dans son ensemble.

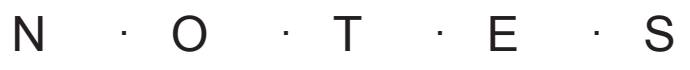

1. François Mitterrand, Rapport «Technologie, emploi et croissance », Sommet de Versailles, 5 juin 1982. http://discours.vie-publique.fr/notices/827078700. html.

2. Y. Stourdzé, Pour une poignée d'électrons : Pouvoir et communication, Paris, Fayard, 1987.

3. B. Chamak, Le Groupe des Dix ou les avatars des rapports entre science et politique, Paris, Les Éditions du Rocher, 1997.

4. Claude Alphandéry, ancien résistant, était le président de la Banque de Construction et des Travaux Publics de 1964 à 1980. Animateur d'un cercle de réflexion, le Club Jean Moulin de 1959 à 1965, il a participé dans les années 1970 au club Échanges et projets, fondé par Jacques Delors. Il a signé un appel en faveur du candidat François Mitterrand pour la présidentielle de 1974.

5. Entretien avec Jacques Robin le 6 mars 1996. Jacques Robin, médecin, directeur général du laboratoire pharmaceutique Midy (future entreprise Sanofi) dans les années 1970, avait rencontré Robert Buron au Mouvement socialiste pour les États-Unis d'Europe. Tous deux ont été à l'initiative de la création du Groupe des Dix, avec le sociologue Edgar Morin et le biologiste Henri Laborit. L'économiste René Passet et Michel Rocard ont également fait partie du Groupe des Dix.

6. Entretien avec Jacques Robin le 6 mars 1996.

7. Entretien avec Jacques Robin le 10 août 1995.

8. Entretien avec Jacques Robin le 6 mars 1996.

9. L'Institut Auguste Comte, créé pour l'étude des sciences de l'action, a été fondé "sur l'idée qu'un enseignement de haut niveau permettrait de sensibiliser les responsables de l'administration et de l'industrie aux conséquences économiques, sociales, 
internationales et environnementales des grands projets industriels, technologiques, et d'aménagement. » (Annexe $\mathrm{n}^{\circ} 2$ du rapport sur le CESTA rédigée par Joël de Rosnay, décembre 1981, Archives nationales, RE 558, liasse1, p. 98). Le CESTA était conçu comme l'antithèse d'un centre élitiste comme l'Institut Auguste Comte, qui était décrit ainsi dans cette même annexe 2 du rapport sur le CESTA: « À partir d'une pédagogie pluridisciplinaire liant la réflexion et l'action, l'Institut Auguste Comte concentre pendant trois ans ses moyens sur la formation d'un nombre réduit de personnes. Cette formation est fondée sur une conception individualiste, au détriment d'une sensibilisation d'un public plus large et d'une production de connaissances résultant de recherches propres. » Le Premier ministre en a suspendu les activités en août 1982 et l'institut a été dissous en octobre 1982.

10. J. de Rosnay, Rapport au Premier ministre : $C E S$ TA : propositions d'organisation, de fonctionnement, et de mise en cuvre, 23 décembre 1981, Archives nationales, RE 558, liasse1, préambule p. 2.

11. Ibid. résumé p. 2

12. Ibid. p. 26.

13. Archives nationales RE 160/22.

14. Technologie, Croissance, Emploi, La Documentation française, collection des rapports officiels, 1983. 15. Ibid.

16. Annexe 7 de la thèse de Jean-Paul Karsenty, Analyse socio-économique de la coopération scientifique et technologique européenne - genèse et ambition d'Eurêka. Thèse de doctorat en sciences économiques, Université Paris I Sorbonne, 1987.

17. Thèse de J.P. Karsenty, op. cit.

18. L'initiative de défense stratégique (IDS) était présentée comme un moyen de préserver l'espèce humaine de la guerre nucléaire grâce à l'élaboration d'un bouclier anti-missile. L'IDS avait été proposée par Ronald Reagan pour « contrecarrer la terrible menace que les missiles soviétiques font peser sur nous », discours du 23 mars 1983. Le 26 mars 1985, le département américain de la Défense avait sollicité par lettre tous les chefs d'État afin qu'ils participent à cette initiative. Cf. B. Chamak, 1997, op. cit. et P. Chaput, P., La France face à l'initiative de Défense Stratégique de Ronald Reagan (1983-1986), 2013, Paris, Édition l'Harmattan.

19. Entretien avec J.P. Karsenty, 13 mai 1996.

20. Thèse de J.P. Karsenty (1987), op.cit., p. 213.

21. George Saunier, «Eurêka : un projet industriel pour l'Europe, une réponse à un défi stratégique », in Journal of European Integration History, vol.12, $\mathrm{n}^{\circ} 2$, 2006, pp. 57-74.

22. La Charte d'Hanovre, Annexe 16 de la thèse de J.P. Karsenty, op.cit., p. 215.

23. Archives nationales, RE 295, liasse 1.

24. B. Chamak, Étude de la construction d'un nouveau domaine : les sciences cognitives. Thèse en épistémologie et histoire des sciences de l'université Paris VII, 1997.

25. Archives nationales, RE 297, liasse 1.

26. Jean-Paul Gillet fut inspecteur à la Direction Générale des Télécommunications.

27. Archives nationales RE 297, liasse 2.

28. Entretien avec Michel Feldmann, 9 mai 1996.

29. Cf. B. Chamak, Thèse, op. cit. ; B. Chamak "The Emergence of Cognitive Science in France: A comparison with the USA", in Social Studies of Science, vol.29, n5, 1999, pp. 643-684; « Les sciences cognitives en France », in La revue pour l'Histoire du CNRS, n 10, 2004, pp. 4-15; «Modèles de la pensée : quels enjeux pour les chercheurs en sciences cognitives? », in Intellectica, 39 (2), 2004, pp. 79-105.

30. F. Antomarchi, A. Castiel, E. Seyden, Pense... machine: Pour comprendre l'intelligence artificielle, Paris, éditions CESTA, 1986.

31. En 1987, le CREA sera rebaptisé « Centre de Re- 
cherche en Epistémologie Appliquée ».

32. Archives nationales, RE 297, liasse 3.

33. Journal Officiel du 18 novembre 1987.

34. Archives nationales, RE 287, liasse1.

35. B. Chamak, Thèse, op. cit. ; B. Chamak, "The Emergence of Cognitive Science in France: A comparison with the USA", op.cit.

36. Daniel Béland, «Idées, institutions politiques et production de l'expertise : une perspective comparative sur le rôle des think tanks au Canada et aux États-Unis », in Quaderni, n70, Paris, Automne 2009, pp. 39-48.

37. Société de savants. Cf. C. Webster, "New light on the Invisible College: the social relations of English science in the mid-seventeenth century", in Transactions of the Royal Historical Society, vol. 24, 1974, pp. 19-42.

38. L. Sfez, Technique et idéologie. Un enjeu de pouvoir, Paris, Le Seuil, 2002.

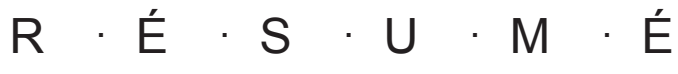

Le Centre d'Étude des Systèmes et Technologies Avancées (CESTA), dirigé par Yves Stourdzé de 1982 à 1986, fut un véritable organisme de conseil de la présidence de la République mais son histoire est assez peu connue. Ce centre a été conçu comme un observatoire du développement technologique, un support à la décision, un carrefour d'animation et d'information sur les technologies de pointe. Cet article vise à retracer les réalisations de cet organisme d'État qui a été dissous en 1987. D'abord impliqué dans l'animation et le suivi du groupe de travail « Technologie, Croissance, Emploi » (TCE), créé au lendemain du Sommet de Versailles, il élabora le projet de coopération technologique européenne : EUREKA. En réponse à la sollicitation en mars 1985 par le département américain de la Défense à participer à l'Initiative de Défense Stratégique (IDS), ou « guerre des étoiles », lancé en mars 1983 par Ronald Reagan, François Mitterrand proposa un projet de coopération européenne orienté vers la recherche civile et les innovations technologiques. Entre mars et juin 1985, Yves Stourdzé et son équipe rédigèrent le livre blanc, La Renaissance technologique de l'Europe, dans le cadre du lancement du programme EUREKA. Dans cet article, je traiterai : 1) de la préhistoire du CESTA et du rôle du Groupe des Dix et de Jacques Attali ; 2) de l'implication d'Yves Stourdzé et du CESTA dans l'animation, et le suivi du groupe de travail TCE et la conception du projet EUREKA ; 3) du rôle du CESTA dans le développement des sciences cognitives en France.

\section{Abstract}

Yves Stourdzé and CESTA achievements

CESTA (Centre d'Études des Systèmes et Technologies Avancées), headed by Yves Stourdzé from 1982 
to 1986 , was an advisory body to the French Office of the President, yet its history is not widely known. The centre was intended to monitor technological developments, to aid in decision-making, and to facilitate the exchange of information about advanced technologies. This article sets out to review the achievements of this government body, which was dissolved in 1987. Involved at the outset in facilitating and evaluating the results achieved by the working group "Technology, Grows and Employment", which was created following the Versailles summit conference, it developed the European project of technological cooperation named EUREKA. In response to the U.S. Defense Department's invitation in March 1985 to take part in the Strategic Defense Initiative (SDI), or "Star Wars" program, launched in March 1983 by Ronald Reagan, François Mitterrand suggested setting up a collaborative European project oriented towards civilian research and technological innovation. Between March and June 1985, Yves Stourdzé and his team drafted a white paper, La Renaissance Technologique de l'Europe, linked to the launching of the EUREKA programme. In this article we will examine 1) the run-up to CESTA and the role of the Group of Ten and Jacques Attali, 2) the involvement of Yves Stourdzé and CESTA in the activities and results of the TCE working group and in the conception of the EUREKA project, and 3) the role of CESTA in the development of cognitive science in France. 